\section{THERAPEUTISCHES KLETTERN}

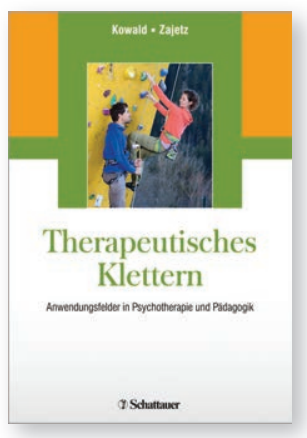

Viele gute Informationen $\rightarrow$ Titel und Werbetext dieses Fachbuchs versprechen praktische Tipps für die Umsetzung eines therapeutischen Klettertrainings. Und das Versprechen hält das Buch. Die Autoren beide aus den Bereichen Sozialpädagogik, Psychotherapie, Psychologie und Sportwissenschaften - sind mit großer Fachkompetenz an das Werk herangegangen und haben es mit umfangreichen psychologischen, sozialpädagogischen und medizinischen Inhalten ausgestattet. Diese vermitteln dem Leser ein breites Basiswissen. Bei den psychologischen und sozialpädagogischen Inhalten erwähnen die Autoren auch ergotherapeutische Bereiche wie
Wahrnehmung, Motorik und Konzentration. Hier gehen sie jedoch sehr psychologisch an die Themen heran. Hilfreich wäre ein therapeutischer Leitfaden mit Angaben zu einem Trainings- oder Therapieaufbau gewesen.

Ein Buch mit umfangreichen, hochwertigen Informationen und vielen praktischen Übungsbeispielen. Es würde sich auch als Nachschlagewerk eignen, wenn es etwas übersichtlicher gestaltet wäre. Der ergo- und physiotherapeutische Bezug beim therapeutischen Klettern kommt aus meiner Sicht etwas zu kurz.

Patricia Willikonsky, Ergotherapeutin und Outdoortherapeutin aus Stuttgart

A.-C. Kowald, A. K. Zajetz (Hrsg.)

Therapeutisches Klettern

Schattauer Verlag 2015, 353 S., 39,99€

ISBN 978-3-7945-2968-1

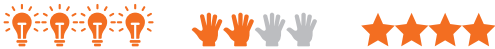

\section{EVOLUTIONSPÄDAGOGIK}

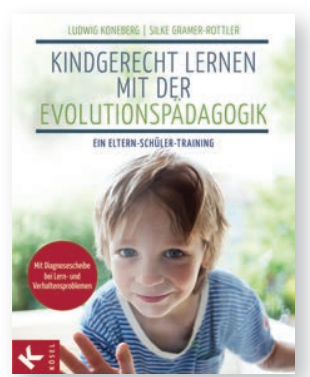

Einseitige Sichtweise $\rightarrow$ Zwei Pädagogen üben in ihrem neuesten Buch eine undifferenzierte Pauschalkritik am bestehenden Schulsystem und der heutigen Pädagogik. Sie setzen Letzterer eine neue Spielart der uralten (angeblich) natürlichen, weisungslosen Pädagogik entgegen. Die Autoren wünschen eine Veränderung von der Anpassungs- hin zur Entfaltungspädagogik, die nach ihrer Meinung auf der Evolutionslehre beruhen soll. Nach dieser Lehre durchläuft jeder Mensch in seiner Entwicklung die Stufen der Evolution (Fisch, Amphibie, Reptil, Säugetier usw.). Jeder Stufe ordnen die Autoren Eigenschaften zu, so dem Fisch das Urvertrauen. Sie halten die momentane Lehre und Beurteilung von Schülern für defizitorientiert und fordern ein Bildungssystem, in dem Lernen nach Interesse und Begabung erfolgt. Treten Probleme auf, müsse man diese nur auf der dem Buch beigefügten „Diagnosescheibe“ suchen und die zugeordnete Bewegungsübung durchführen.
Der Ansatz, demzufolge Ergotherapeuten übrigens auf der Reptil-Ebene (Körpersicherheit/Kraft) arbeiten, ist eine grobe Versimplifizierung komplexer Sachverhalte. Den sich häufig wiederholenden Ausführungen fehlt jede stringente Gedankenentwicklung. Dieser als Handbuch deklarierte Aufruf zur Umkehr wartet mit dem Versprechen einer schnellen, einfachen Abhilfe für alle pädagogischen Probleme auf, selbst für die zukünftigen. Die Autoren vermitteln ihr Ansinnen in meist reduzierter Sprache, wohl in der Annahme, die Betroffenen damit besser erreichen zu können.

Das Buch ist aus einseitiger Sicht geschrieben und wird dem Anspruch, ein Eltern-Schüler-Training zu bieten, in keiner Weise gerecht. Susanne Harder-Sdzuj, BcOT (NL), Ergotherapeutin aus Greifswald

L. Koneberg, S. Gramer-Rottler

Kindgerecht lernen mit der

Evolutionspädagogik

Kösel-Verlag 2014, 112 S., 15,99€

ISBN 978-3-466-31025-8

\section{LESEREMPFEHLUNG}

Mut machend $\rightarrow$ Bettina Unger ist noch jung, als sie an Multipler Sklerose erkrankt. In ihrem Buch „Du rockst, ich roll“ beschreibt sie, wie sie nach anfänglicher Verzweiflung lernt, mit dieser Krankheit umzugehen. Voller Humor und messerscharf beobachtend schildert sie die verschiedenen Phasen der Erkrankung. Sie beschreibt, wie sich ihr Körper im Laufe der Jahre verändert und wie sie ständig neue Situationen bewältigen muss. Trotz der zahlreichen Niederschläge meistert sie ihr Leben mit Bravour, und dafür hat sie meinen allergrößten Respekt!

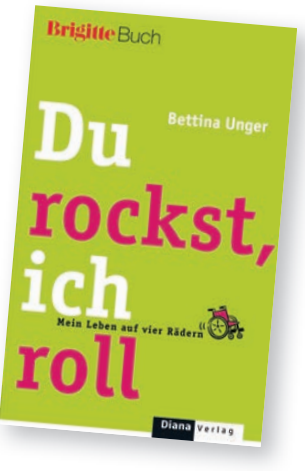

Ich habe das Buch schon mehreren Patienten, Kollegen sowie Schülern ausgeliehen, da es zeigt, wie heftig einem Menschen das Leben mitspielen kann und dieser dennoch die Hoffnung nicht verliert.

Das Buch macht Betroffenen und Angehörigen Mut. Auch Therapeuten kann ich es empfehlen, da es ihnen hilft, sich in Menschen mit Multipler Sklerose einzufühlen und die Krankheit besser kennenzulernen. Absolut empfehlenswert!

Rebecca Schneider, Physiotherapeutin aus Würzburg

B. Unger

„Du rockst, ich roll“

Diana Verlag 2012, eBook, 13,99€

ISBN 978-3-641-07335-0

\section{LESEREMPFEHLUNG}

Wollen Sie Ihren Kolleginnen und Kollegen ein Buch empfehlen? Schicken Sie Ihren Vorschlag an: ergopraxis@thieme.de.

Wir belohnen die besten Tipps mit einem Platz in ergopraxis und einem 20-Euro-Thieme-Buchgutschein! 